\title{
Strenge Blutzuckereinstellung senkt Sterblichkeit nicht
}

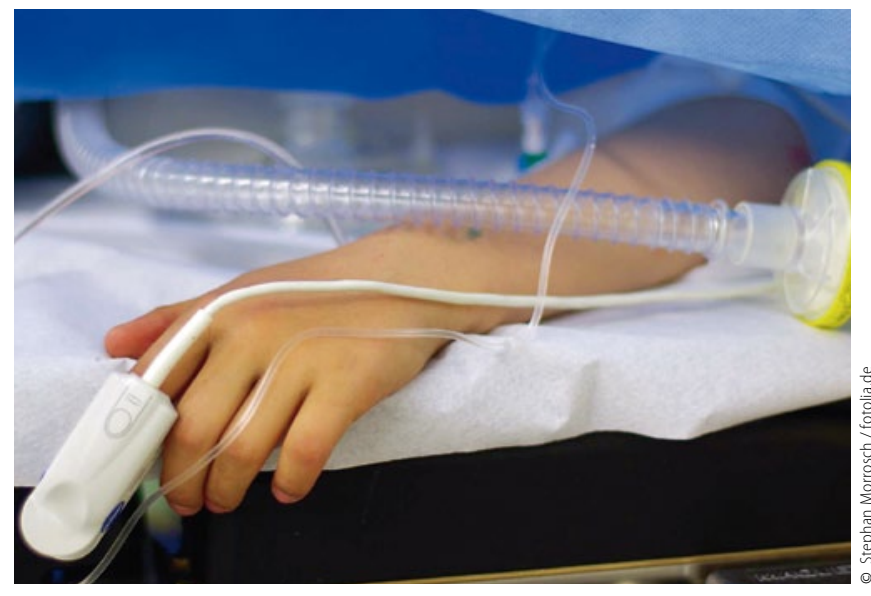

Eine strenge Blutzuckereinstellung beeinflusst zudem weder die Infektionsrate, die Aufenthaltsdauer in der Klinik oder das Auftreten von Organversagen nach einer Herzoperation bei Kindern.

Fragestellung: Verbessert eine strenge Blutzuckereinstellung die Morbidität nach pädiatrisch herzchirurgischen Eingriffen bei nicht diabetischen Kleinkindern?

Hintergrund: Einige Studien zeigen, dass die strenge Blutzuckereinstellung mit Insulin das Outcome bei Erwachsenen verbessert, die sich einem herzchirurgischen Eingriff unterzogen haben. Für Kinder, die intensivmedizinisch behandlungspflichtig sind und ein Risiko für hyperinsulinämische Hypoglykämie haben, liegen keine Daten vor.

Patienten und Methodik: Die Studie wurde prospektiv, randomisiert an zwei Zentren realisiert. 980 Kinder im Alter zwischen 0 und 36 Monaten erhielten auf der intensiv-medizinischen Abteilung (ICU) entweder eine strenge Blutzuckerkontrolle (mithilfe eines Insulin-Dosis Algorithmus, der Blutzuckerwerte von

$80-110 \mathrm{mg} / \mathrm{dl}$ [4,4 bis 6,1 mmol/l] anstrebte) oder eine Standardbehandlung, die keine Blutglukosezielwerte angab. Die kontinuierliche Blutzuckermessung sollte einen Anhalt für die Frequenz der Blutzuckermessungen geben und Hypoglykämien erkennen lassen.

Der primäre Endpunkt war die Rate an Behandlungs-assoziierten Infektionen auf der kardiologischen ICU. Sekundäre Endpunkte waren Mortalität, Länge des Aufenthalts auf der Intensivstation, Organversagen und Hypoglykämien.

Ergebnisse: 444 von 490 Kindern (Interventionsgruppe: 91\%) bekamen Insulin, 9 der 490 Kinder erhielten eine Standardbehandlung (2\%). Die Normoglykämie erreichten die Kinder mit der strengen Blutzuckerkontrolle früher als diejenigen mit der Standardbehandlung (6 vs. 16 Stunden, $\mathrm{p}<0,001$ ). Diese konnte zu einem Großteil über die Phase der intensiv-medizinischen Behandlung aufrecht erhalten werden (50 vs. 33\%, p <0,001).

Die strengere Blutzuckereinstellung zeigte keine signifikant verminderte Rate an Behandlungs-assoziierten Infektionen (8,6 vs. 9,9 pro 1000 Patienten-Tage, $p=0,67$ ). Die sekundären Endpunkte unterschieden sich nicht signifikant zwischen den Gruppen. Die strenge Blutzuckerkontrolle bot auch keine Vorteil in den Hochrisiko-Gruppen. Nur 3\% der Patienten mit strenger Blutzuckerkontrolle litten an schweren Hypoglykämien (Blutglukose unter $40 \mathrm{mg} / \mathrm{dl}$ [2,2 $\mathrm{mmol} / \mathrm{l}])$.

Schlussfolgerungen: Eine strenge Blutzuckerkontrolle nach kardiochirurgischen Eingriffen beeinflusst nicht signifikant die Infektionsrate, Mortalität, Dauer des Aufenthaltes oder das Auftreten von Organversagen, wenn sie mit der Standardbehandlung verglichen wird.

Agus MS, Steil GM, Wypij D, et al.; SPECS Study Investigators; Boston, USA. Tight glycemic control versus standard care after pediatric cardiac surgery. $\mathrm{N}$ Engl J Med 2012;367(13):1208-19.

\section{- Kommentar von PD Dr. med. Nanette C. Schloot}

\section{Auch für Kinder kein Vorteil}

Nachdem für Erwachsene auf Intensivstation aufgrund von mehreren Studienergebnissen keine niedrige Blutglukoseeinstellung mehr empfohlen wird, wurde in einer Studie von Vlasselaers aus dem Jahr 2009 ein Vorteil bei Kindern auf Intensivstation gefunden. Die aktuelle Studie hat die Kriterien für die Blutzuckereinstellung etwas höher gesetzt $(80-110 \mathrm{mg} / \mathrm{dl}$ vs. 70-100 mg/dl). Sie findet jedoch keinerlei Vorteil für die Kinder nach herzchirurgischen Eingriffen.

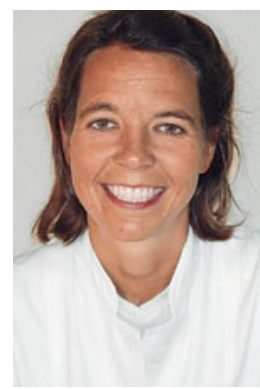

PD Dr. med. Nanette C. Schloot

Gastwissenschaftlerin

Deutsches Diabetes-Zentrum an der Heinrich-Heine Universität Düsseldorf Institut für Klinische Diabetologie Auf'm Hennekamp 54 40225 Düsseldorf schloot@ddz.uni-duesseldorf.de 